\title{
Serious Bleeding Events due to Warfarin and Antibiotic Co- prescription In a Cohort of Veterans
}

\author{
Michael A. Lane, MD MSc ${ }^{1}$, Angelique Zeringue, $\mathbf{M S}^{1,2}$, and Jay R. McDonald, MD ${ }^{2,1}$ \\ ${ }^{1}$ Washington University, St. Louis, MO \\ ${ }^{2}$ St. Louis Veterans Affairs Medical Center
}

\begin{abstract}
Background-Antibiotics may interact with warfarin, increasing the risk for significant bleeding events.

Methods-Retrospective cohort study of veterans prescribed warfarin for $\geq 30$ days without interruption through the VA between October 1, 2002 and September 1, 2008. Antibiotics considered to be high-risk for interaction with warfarin include: trimethoprim/sulfamethoxazole (TMP/SMX), ciprofloxacin, levofloxacin, metronidazole, fluiconazole, azithromycin, and clarithromycin. Low-risk antibiotics include: clindamycin and cephalexin. Risk of bleeding event within 30 days of antibiotic exposure was measured using Cox proportional hazards regression, adjusted for demographic characteristics, comorbid conditions and receipt of other medications interacting with warfarin.
\end{abstract}

\begin{abstract}
Results-A total of 22,272 patients met inclusion criteria with 14,078 and 8,194 receiving highand low-risk antibiotics, respectively. There were 93 and 36 bleeding events in the high- and lowrisk groups, respectively. Receipt of a high-risk antibiotic (HR 1.48, 95\% CI 1.00-2.19) and azithromycin (HR 1.93, 95\% CI 1.13-3.30) were associated with increased risk of bleeding as a primary diagnosis. TMP/SMX (HR 2.09, 95\% CI 1.45-3.02), ciprofloxacin (HR 1.87, 95\% CI 1.42-2.50), levofloxacin (HR 1.77, 95\% CI 1.22-2.50), azithromycin (HR 1.64, 95\% CI 1.16-2.33), and clarithromycin (HR 2.40, 95\% CI 1.16-4.94) were associated with serious bleeding as a primary or secondary diagnosis. INR alterations were common; $9.7 \%$ of patients prescribed fluconazole had INR value $>6$. Patients who had INR performed 3-14 days of co-prescription were at a decrease risk of serious bleeding (HR 0.61, 95\% CI 0.42-0.88).
\end{abstract}

Conclusions-Warfarin users who are prescribed high-risk antibiotics are at higher risk for serious bleeding events. Early INR evaluation may mitigate this risk.

(C) 2014 Elsevier Inc. All rights reserved.

Corresponding Author \& Requests for Reprints: Michael A. Lane, MD, MSc, 660 S. Euclid Ave, Campus Box 8051, St. Louis, MO 63110, Phone: 314-454-8293, Fax: 314-454-5392, mlane@ dom.wustl.edu.

Conflict of Interest: The authors have no conflicts of interest to report.

Authorship Statement: All authors had access to the data and participated in writing this manuscript.

Publisher's Disclaimer: This is a PDF file of an unedited manuscript that has been accepted for publication. As a service to our customers we are providing this early version of the manuscript. The manuscript will undergo copyediting, typesetting, and review of the resulting proof before it is published in its final citable form. Please note that during the production process errors may be discovered which could affect the content, and all legal disclaimers that apply to the journal pertain. 


\section{Keywords}

Warfarin; antibiotics; drug interactions; patient safety; pharmacoepidemiology; bleeding

\section{Introduction}

Warfarin is a widely used anticoagulant with indications for the prevention and treatment of thromboembolic events in patients with atrial fibrillation ${ }^{1-4}$, mechanical heart valves ${ }^{5}$ and venous thromboembolisms. ${ }^{6}$ Warfarin has a narrow therapeutic index, requiring frequent laboratory monitoring to prevent life-threatening complications due to under- and overanticoagulation. ${ }^{7,8}$

Warfarin produces its anticoagulant effect by inhibiting the vitamin K-dependent activation of clotting factors II, VII, IX and X. ${ }^{9}$ A wide variety of factors including genetic factors ${ }^{10}, 11$, dietary factors ${ }^{12}$, drug interactions ${ }^{13}$ and comorbidities ${ }^{7}$ can produce significant variability in an individual's dose-response to warfarin. Co-prescribed medications may alter the metabolism of warfarin by inducing or inhibiting cytochrome P450-2C9, resulting in a decrease or increase in anticoagulant effect. ${ }^{14}$ Interactions between warfarin and antibiotics including trimethoprim/sulfamethoxazole (TMP/SMX), ${ }^{15}, 16$ metronidazole,${ }^{17}$ fluconazole, ${ }^{18,19}$ ciprofloxacin $^{20}$, levofloxacin ${ }^{15}$, azithromycin ${ }^{21,22}$, and clarithromycin ${ }^{23}$ have been described. In addition to interacting with warfarin via cytochrome $\mathrm{P} 450-2 \mathrm{C} 9$, these antibiotics may also eliminate vitamin K-producing bacteria in the intestines to further alter INR. ${ }^{24,25}$ Conversely, cephalexin and clindamycin are believed to have only minimal interactions with warfarin. Prior studies have demonstrated that warfarin users are commonly prescribed antibiotics with known interactions. ${ }^{26}$

In this study, we used the Department of Veterans' Affairs (VA) national databases to characterize the risk of serious bleeding events that require hospitalization among warfarin users who receive antibiotics. We have utilized VA pharmacy, administrative and laboratory databases to identify risk factors for serious bleeding events.

\section{Methods}

This study was approved by the institutional review boards of the participating institutions.

\section{Data Sources}

Inpatient and outpatient International Classification of Diseases, Version 9, Clinical Modification (ICD-9-CM) diagnosis codes, encounter data and patient demographic data were obtained from the VA's Austin Information Technology Center, a repository for VA administrative data. Pharmacy data for all study subjects, including both inpatient and outpatient medications, were obtained from the VA's Pharmacy Benefits Management program. 


\section{Study Population}

The study included all veterans who were prescribed warfarin for $\geq 30$ days without interruption from a VA between October 1, 2002 and September 1, 2008. Subjects were required to have had $\geq 2$ international normalized ratio (INR) lab results while receiving warfarin to demonstrate utilization of the VA for ongoing healthcare.

\section{Subject-time in cohort}

Subject-time began 30 days after the date of the first warfarin prescription, in order to establish a stable warfarin regimen and exclude the initial dose-finding period during which bleeding risk may be elevated. Since prescription length for warfarin is 30 days, subject-time continued while warfarin prescriptions continued, allowing lapses of up to 30 days between subscriptions. Subject-time ended 30 days after the last projected warfarin pill (last prescription date plus duration of prescription). Subjects contributed more than one continuous treatment course of warfarin if they were treated with warfarin on multiple occasions with greater than 30 day lapse during the study period.

\section{Antibiotic exposure}

Subjects were considered to be receiving an antibiotic from the date of prescription plus the number of days prescribed. Antibiotic prescriptions lasting less than 3 days were excluded. Antibiotics were grouped based upon what is known about their degree of interaction with warfarin. Antibiotics which are known to interact in a way which may increase bleeding risk were considered "high-risk." High-risk antibiotics included trimethoprim/sulfamethoxazole (TMP/SMX), ciprofloxacin, levofloxacin, metronidazole, fluconazole, azithromycin and clarithromycin. Low-risk antibiotics included clindamycin and cephalexin. For patients receiving two high-risk antibiotics, outcomes were attributed to each medication equally. Outcomes that occurred among patients receiving high- and low-risk antibiotics wereattributed to the high-risk antibiotic.

\section{Primary outcome}

The primary outcome was hospitalization for serious bleeding event. A hospitalization was considered to be caused by a serious bleeding event if an ICD-9-CM code found in Appendix 1 occurred in the field designating primary reason for hospitalization. ICD-9-CM codes for serious bleeding event are a modification of a previously described group of codes. ${ }^{27} \mathrm{~A}$ bleeding event that occurred during subject-time was attributed to an antibiotic if it occurred within 30 days of the last prescribed dose of the antibiotic.

\section{Secondary outcomes}

Secondary outcomes included elevations in INR $>4$, and serious bleeding events coded in secondary diagnosis fields of a hospitalization.

\section{Data analysis}

For descriptive and bivariate analysis, chi-square test was used for dichotomous variables. Continuous variables were analyzed using student's t-test. A two-sided p-value of $\leq 0.05$ was considered statistically significant. Risk of outcomes was described using hazard rations and 
95\% confidence intervals. Cox proportional hazards regression was performed for time to event analysis. Regression models were adjusted for age, gender, race, comorbid diagnosis, indication for anticoagulation and coprescription of other medications known to interact with warfarin. Each drug was modeled separately and adjusted as described. Because patients living further from a VA hospital are more likely to seek care at a non-VA facility in case of emergency, the model was adjusted for proximity to the nearest VA hospital based upon zip code data. Patients residing in zip codes where the mean distance was $>20$ miles from the nearest VA hospital were considered to be distant to a VA hospital. Maximum INR values within 30 days of antibiotic exposure were analyzed by antibiotic. During data analysis, INR values from two VA hospitals were found to have a disproportionate number of values $>12$. After closer evaluation, these values likely represented a systematic error (entry of prothrombin times instead of INR into the INR field), and reported INR values from these facilities were excluded from analysis. All analyses were performed using SAS software version 6.12 (SAS Institute, Cary, NC).

\section{Validation}

Fifty patients who were hospitalized for a bleeding event and 50 patients not hospitalized for a bleeding event at a single center during the study period were randomly identified by ICD-9-CM codes. All medical records, including admission histories, discharge summaries, and daily notes were reviewed by two physicians (M.A.L and J.R.M). Hospitalization was classified as "for a bleeding event" or "not for a bleeding event" by each reviewer based on their interpretations of primary reason for hospitalization. Consensus was achieved between the 2 reviewers in all cases. Adjusted sensitivity and specificity were calculated by extrapolating the validation results over the entire study population.

\section{Results}

There were a total of 22,272 veterans on warfarin for $\geq 30$ days who were also prescribed an included antibiotic during the study period (Table 1). There were 8,194 patients who received a low-risk antibiotic and 14,078 patients who received a high-risk antibiotic. Patients receiving a high-risk antibiotic were slightly older and were more likely to have a malignancy or chronic lung disease. Diabetes mellitus, hypertension and alcohol dependence were slightly less common among those receiving a high-risk antibiotic. Those receiving a high-risk antibiotic were less likely to be receiving anticoagulation for heart valve replacement compared to warfarin users who received low-risk antibiotics. Patients prescribed a high-risk antibiotic were also more frequently receiving other medications known to interact with warfarin when compared to those receiving a low-risk antibiotic. More patients receiving a high-risk antibiotic lived $>20$ miles from the nearest VA when compared to those receiving low-risk antibiotics. Additionally, more patients receiving a high-risk antibiotic had an INR test within 3-14 days of antibiotic prescription when compared to those who were prescribed a low-risk antibiotic.

There were 36 and 93 bleeding events among patients receiving a low- and high-risk antibiotic, respectively (Table 2). The demographic characteristics between those who had a serious bleeding event and those that did not were largely similar. Malignancy (HR 1.89, 
95\% CI 1.33-2.70) and renal failure (HR 2.53, 95\% CI 1.74-3.68) were associated with an increased risk of serious bleeding events. Co-prescription of other interacting medications increased the risk of serious bleeding events (HR 2.29, 95\% CI 1.01-5.19). Early INR evaluation, occurring within 3-14 days of antibiotic co-prescription, reduced the risk of serious bleeding events (HR 0.61, 95\% CI 0.42-0.88). Receipt of a high-risk antibiotic also increased the risk of serious bleeding when compared to receipt of a low risk antibiotic (HR $1.48,95 \%$ CI 1.00-2.19). Of the individual high-risk antibiotics, azithromycin was associated with an increased risk of serious bleeding event when compared to the low-risk antibiotics (HR 1.93, 95\% CI 1.13-3.30). When ICD-9-CM codes for serious bleeding events in either the primary or secondary diagnosis position were considered, diabetes mellitus (HR 1.25, 95\% CI 1.01-1.56), malignancy (HR 1.56, 95\% CI 1.27-1.97) and renal failure (HR 2.07, 95\% CI 1.64-2.60) were associated with an increased risk for serious bleeding events. Early INR evaluation was associated with a similar reduction in risk of bleeding as seen in the primary outcome measure. Gastrointestinal bleeding events accounted for $57 \%$ of all serious bleeding events among patients receiving high-risk antibiotics, followed by bleeding in the genitourinary system (24.7\%).

In the secondary outcomes analysis examining ICD-9-CM codes for serious bleeding seen in either primary or secondary coding position, TMP/SMX (HR 2.09, 95\% CI 1.45-3.02), ciprofloxacin (HR 1.87, 95\% CI 1.42-2.50), levofloxacin (HR 1.77, 95\% CI 1.22-2.55), azithromycin (HR 1.64, 95\% CI 1.16-2.33) and clarithromycin (HR 2.40, 95\% CI 1.16-4.94) were associated with increased risk of serious bleeding when compared to the low risk antibiotics. Alterations in INR were common among warfarin users receiving antibiotics (Figure 3). Among those prescribed a high-risk antibiotic, 7.8\% had INR elevations $>4-\leq 6$. Among individual antibiotics, metronidazole (10.1\%), fluconazole (13.9\%), and receipt of two or more high-risk antibiotics $(11.1 \%)$ was associated with elevations of INR $>4-\leq 6$. When compared to those prescribed a low-risk antibiotic, significant INR elevations $\geq 6$ were seen in $9.7 \%(\mathrm{p}<.0001)$ and $4.9 \%(\mathrm{p}<.0001)$ of patients receiving fluconazole and metronidazole, respectively. There were no substantial differences in types of bleeding events between antibiotic groups (data not shown).

The medical records of 50 patients identified by ICD-9-CM codes as being hospitalized for a bleeding event and 50 patients not hospitalized for a bleeding event were reviewed. Bleeding events were confirmed in 48 patients, resulting in a positive predictive value of the administrative data to identify a hospitalization for bleeding of $96 \%$. There were 5 hospitalizations for bleeding among the 50 patients identified as not having a bleeding event during the study period. The sensitivity and specificity of the ICD-9 codes to identify a bleeding event was $91 \%$ and $96 \%$, respectively. The kappa was 0.86 , indicating excellent agreement between the code and our gold standard.

\section{Discussion}

In this large cohort of warfarin users in the VA healthcare system, we describe the risk of and risk factors for serious bleeding events following prescription of selected antibiotics. We demonstrated excellent validity of our outcome measure using electronic medical records. We have shown that patients receiving high-risk antibiotics are at increased risk for serious 
bleeding events compared to those receiving low-risk antibiotics. Warfarin users who received azithromycin had nearly twice the risk of hospitalization for serious bleeding compared to low-risk antibiotics. When ICD-9-CM codes for serious bleeding were permitted in the primary or secondary reasons for admission, TMP/SMX (HR 2.09, 95\% CI 1.45 - 3.02), ciprofloxacin (HR 1.87, 95\% CI 1.42 - 2.50), levofloxacin (HR 1.77, 95\% CI 1.22 - 2.55), azithromycin (HR 1.64, 95\% CI 1.16 - 2.33), clarithromycin (HR 2.40, 95\% CI 1.16 - 4.94) all increased the risk for serious bleeding events.

Bleeding events were the primary reason for admission in $0.6 \%$ of all patients included in this study. A study utilizing a 5\% national sample of Medicare beneficiaries with Part D benefits, $2.9 \%$ of warfarin users met the case definition of a serious bleeding event. However, differences in study methodology may account for the difference in frequency of bleeding events. In the Medicare study, $78.7 \%$ all warfarin users who had a bleeding event were not exposed to antibiotics prior to the bleeding event. Our study includes only those patients who are prescribed warfarin and an antibiotic concurrently based on VA pharmacy data. Additionally, this Medicare study includes bleeding events occurring $>60$ days after antibiotic exposure, whereas our study includes those within 30 days of co-prescription. ${ }^{28} \mathrm{~A}$ study of 19,935 new users of coumarin anticoagulants in the Netherlands found a 4 to 7 -fold increased risk of bleeding related to antibiotics. ${ }^{29}$ However, the study did not control for potential confounders. Additionally, patients included in the study were incident users of coumarin anticoagulants. Prior studies have shown significantly higher rates of serious bleeding events in the period immediately after initiation of anticoagulation. ${ }^{30-33} \mathrm{We}$ excluded the early period after initiation in order to estimate the rate of complications during stable long-term use.

We found that exposure to high-risk antibiotics increases the risk of serious bleeding events as primary diagnosis by nearly $50 \%$ when compared to low-risk antibiotics. Azithromycin nearly doubled the risk of serious bleeding event. When primary and secondary diagnosis codes were included, nearly all high-risk antibiotics increased the risk for bleeding. TMP/SMX was associated with a >2-fold increased risk for bleeding. Other studies have also found that TMP/SMX $28,34,35$, fluoroquinolones ${ }^{28}$ and macrolides ${ }^{28}$ significantly increase the risk for serious bleeding. Prior studies have found a significant increased risk of serious bleeding among warfarin users who received fluconazole. ${ }^{28,} 35$ However, only 3 patients who received fluconazole in our study had a bleeding event, and thus we are likely underpowered to show a clinically significant interaction. Likewise, there were only 5 warfarin users who were prescribed metronidazole and had a bleeding event. Infection may cause elevations in INR independent of antibiotic use. ${ }^{36}$ However, our results suggest infection alone does not alter the bleeding risk among warfarin users. All patients in our cohort received antibiotics for an infection, but only those who received a high-risk antibiotic were at increased risk of serious bleeding events.

Anticoagulation intensity, as measured by INR, has been associated with increased risk of serious bleeding. ${ }^{8,37-40}$ Risk of intracranial hemorrhage has been shown to increase significantly at INR values $>3.5 .8,41$ Many studies demonstrating INR alterations after antibiotic administration have been relatively small. Among 27 patients on stable warfarin regimens who were co-prescribed levofloxacin, $11 \%$ had an elevation of INR $\geq 5$. Among 16 
patients prescribed TMP/SMX, 31\% had an elevation of INR $25 .{ }^{15}$ In our study, elevations in INR were seen among all high-risk antibiotics. Notably, $4.9 \%$ of patients receiving metronidazole and $9.7 \%$ of those prescribed fluconazole had a peak INR $>6$, putting these patients at significant risk for harm. Prior studies suggest infection may independently cause elevations in INR. ${ }^{36}$ It is possible some of the alterations in INR are due to the underlying infection. In our multivariate analysis, INR evaluation within 3-14 days of antibiotic coprescription reduced the risk of serious bleeding event by $39 \%$. This finding strongly supports early INR check after coprescription.

Our study has several limitations that may influence the results. This study relies on the accuracy of ICD-9-CM codes to identify bleeding events. Evaluations of the ICD-9-CM codes used in this study have demonstrated the ability to accurately identify serious bleeding events. ${ }^{2742}$ Additionally, our validation demonstrated excellent agreement between the ICD-9-CM code definition and the electronic medical records (kappa $=0.86$ ). However, it is possible that some serious bleeding events were misclassified. In order for us to identify an outcome, a subject must have utilized a VA healthcare facility for their health care needs. We attempted to limit the impact of this by only including patients who have $\geq 2$ INR values checked in the VA system prior to outcome, to indicate utilization of the VA for healthcare services. Additionally, our multivariate models included distance to the nearest VA hospital to control for the higher likelihood of seeking non-VA care for a bleeding event in subjects residing further from the nearest VA facility. In addition to antibiotics, multiple medications interact with warfarin. We included interacting medications as a dichotomous variable in our models, but did not assess the impact of individual medications. However, given the complex nature of these multiple drug interactions, this approach may approximate realworld clinical practice. Additionally, we were unable to assess patient adherence to antibiotic therapy which could alter our estimate of the interaction effect.

Although warfarin is effective in the prevention and treatment of thromboembolic events, its narrow therapeutic index and multiple drug interactions put patients at risk for serious bleeding events. In this study we have used national VA administrative data to demonstrate an increased risk for serious bleeding events among warfarin users who receive high-risk antibiotics. Our findings suggest that when possible, clinicians should choose antibiotics with low potential to interact with warfarin. Our data strongly supports INR monitoring after co-prescription as a means to decrease risk of hospitalization for serious bleeding.

\section{Acknowledgments}

Financial Support: : Dr. Lane has received career development support from the Goldfarb Patient Safety \& Quality Fellowship program and the Barnes-Jewish Hospital Foundation. Dr. Lane was also supported by the Washington University Institute of Clinical and Translational Sciences grants UL1 TR000448 and KL2 TR000450 from the National Center for Advancing Translational Sciences and the KM1 Scholars Program grant KM1CA156708 through the National Cancer Institute (NCI) at the NIH. Dr. Lane has also received Loan Repayment support from L30 AR063363. The career development of Dr. McDonald has been supported by NIH K12RR023249 and KL2RR024994. 


\section{Appendix 1: Current Procedural Terminology (CPT) and International Classification of Diseases, Ninth Revision (ICD-9) Codes Used}

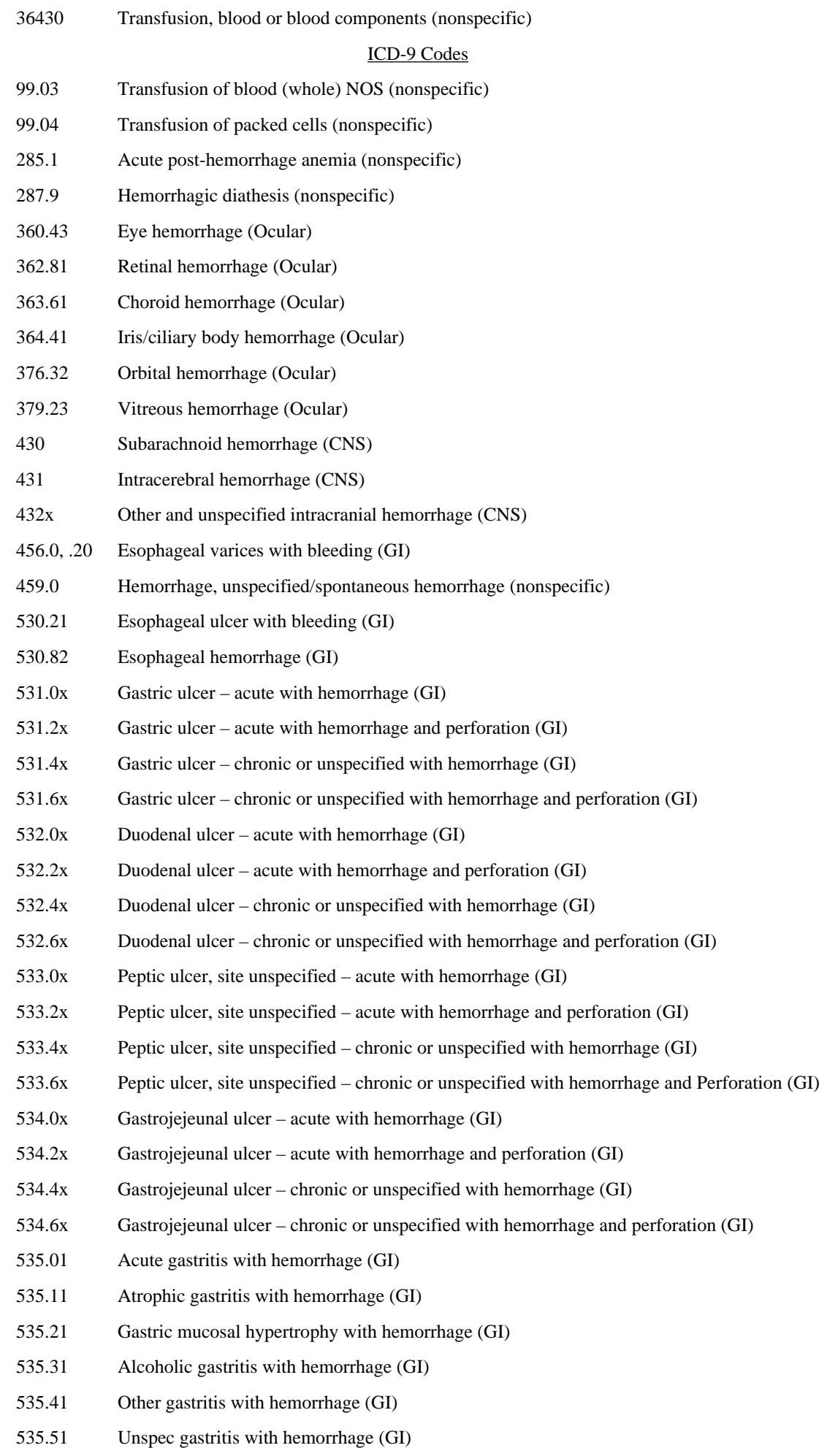




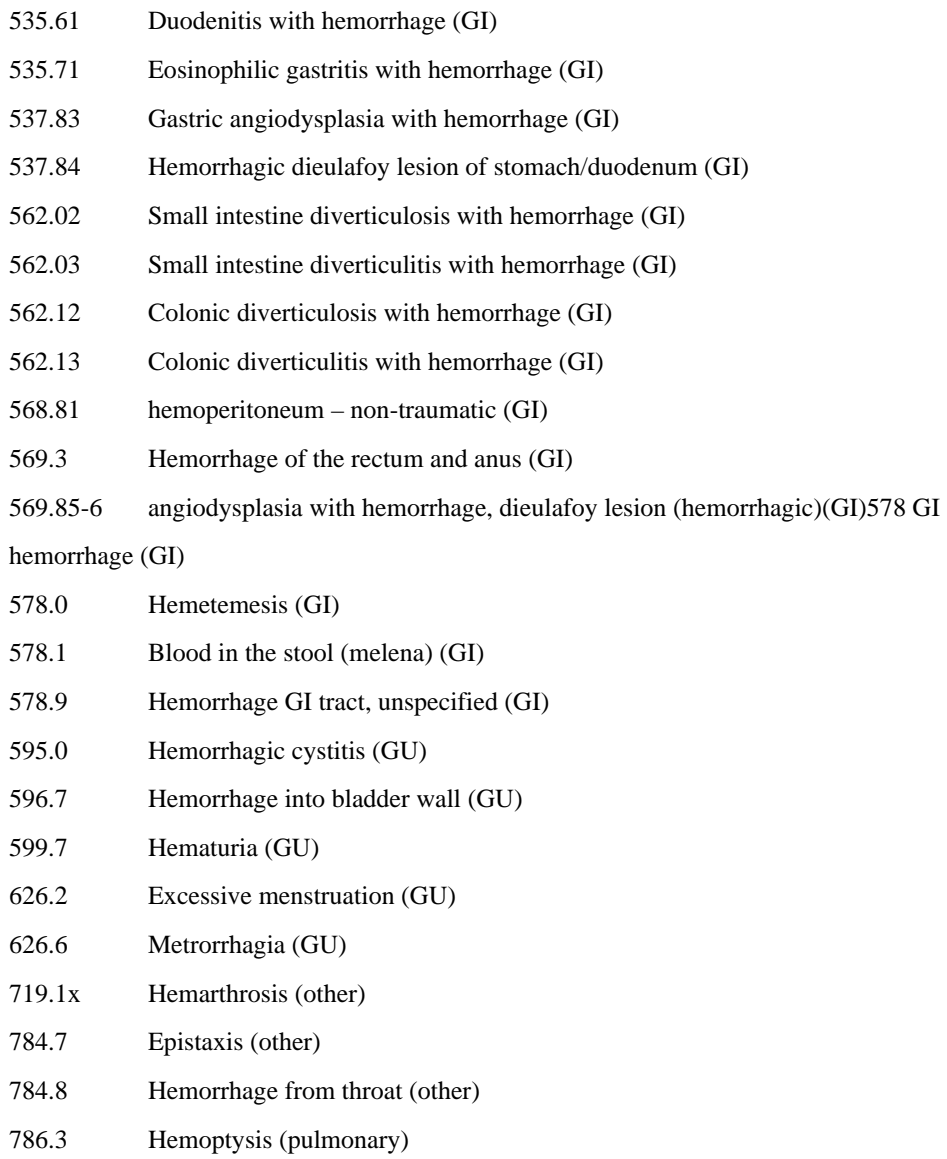

Bleeding event by system:

CNS:

$\begin{array}{ll}430 & \text { Subarachnoid hemorrhage } \\ 431 & \text { Intracerebral hemorrhage } \\ 432 \mathrm{x} & \text { Other and unspecified intracranial hemorrhage } \\ \text { Ocular: } & \\ 360.43 & \text { Eye hemorrhage (Ocular) } \\ 362.81 & \text { Retinal hemorrhage (Ocular) } \\ 363.61 & \text { Choroid hemorrhage (Ocular) } \\ 364.41 & \text { Iris/ciliary body hemorrhage (Ocular) } \\ 376.32 & \text { Orbital hemorrhage (Ocular) } \\ 379.23 & \text { Vitreous hemorrhage (Ocular) } \\ \text { GI: } & \\ 456.0, .20 & \text { Esophageal varices with bleeding (GI) } \\ 530.21 & \text { Esophageal ulcer with bleeding (GI) } \\ 530.82 & \text { Esophageal hemorrhage (GI) } \\ 531.0 \mathrm{x} & \text { Gastric ulcer - acute with hemorrhage (GI) } \\ 531.2 \mathrm{x} & \text { Gastric ulcer - acute with hemorrhage and perforation (GI) } \\ 531.4 \mathrm{x} & \text { Gastric ulcer - chronic or unspecified with hemorrhage (GI) } \\ 531.6 \mathrm{x} & \text { Gastric ulcer - chronic or unspecified with hemorrhage and perforation (GI) } \\ 532.0 \mathrm{x} & \text { Duodenal ulcer - acute with hemorrhage (GI) }\end{array}$




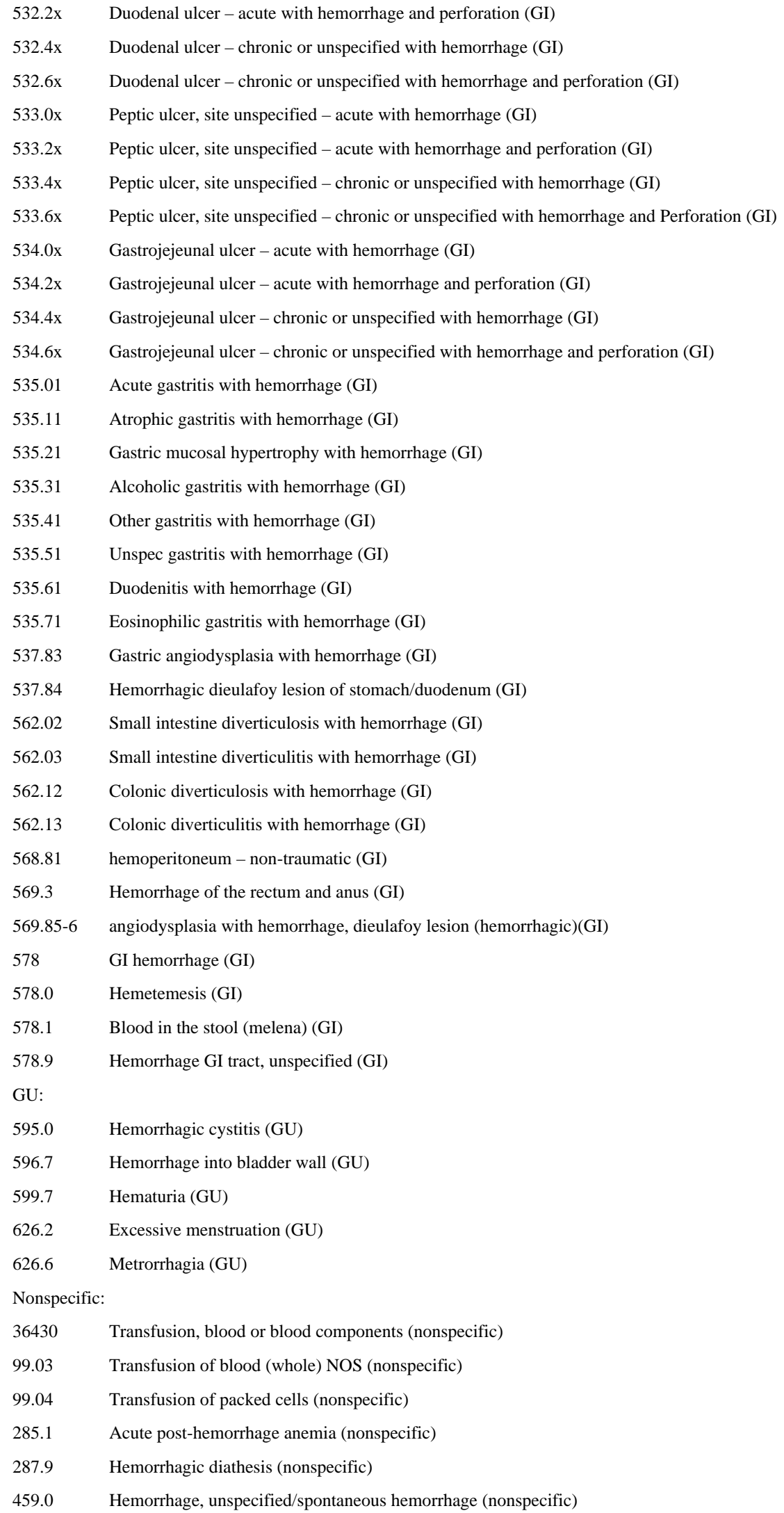




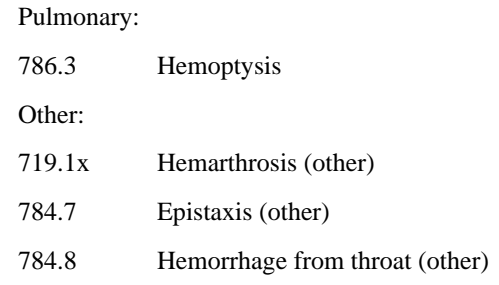

\section{References}

1. The effect of low-dose warfarin on the risk of stroke in patients with nonrheumatic atrial fibrillation. The Boston Area Anticoagulation Trial for Atrial Fibrillation Investigators. N Engl J Med. 1990; 323(22):1505-11. [PubMed: 2233931]

2. Ezekowitz MD, Bridgers SL, James KE, et al. Warfarin in the prevention of stroke associated with nonrheumatic atrial fibrillation. Veterans Affairs Stroke Prevention in Nonrheumatic Atrial Fibrillation Investigators. N Engl J Med. 1992; 327(20):1406-12. [PubMed: 1406859]

3. Stroke Prevention in Atrial Fibrillation Study. Final results. Circulation. 1991; 84(2):527-39. [PubMed: 1860198]

4. Petersen P, Boysen G, Godtfredsen J, et al. Placebo-controlled, randomised trial of warfarin and aspirin for prevention of thromboembolic complications in chronic atrial fibrillation. The Copenhagen AFASAK study. Lancet. 1989; 1(8631):175-9. [PubMed: 2563096]

5. Bonow RO, Carabello BA, Chatterjee K, et al. 2008 Focused update incorporated into the ACC/AHA 2006 guidelines for the management of patients with valvular heart disease. Circulation. 2008; 118(15):e523-661. [PubMed: 18820172]

6. Kearon C, Kahn SR, Agnelli G, et al. Antithrombotic Therapy for Venous Thromboembolic Disease*. Chest. 2008; 133(6 suppl):454S-545S. [PubMed: 18574272]

7. Schulman S, Beyth RJ, Kearon C, Levine MN. Hemorrhagic complications of anticoagulant and thrombolytic treatment: American College of Chest Physicians Evidence-Based Clinical Practice Guidelines (8th Edition). Chest. 2008; 133(6 Suppl):257S-98S. [PubMed: 18574268]

8. Fang MC, Chang Y, Hylek EM, et al. Advanced age, anticoagulation intensity, and risk for intracranial hemorrhage among patients taking warfarin for atrial fibrillation. Ann Intern Med. 2004; 141(10):745-52. [PubMed: 15545674]

9. Hirsh J, Dalen J, Anderson DR, et al. Oral anticoagulants: mechanism of action, clinical effectiveness, and optimal therapeutic range. Chest. 2001; 119(1 Suppl):8S-21S. [PubMed: 11157640]

10. Gage BF, Lesko LJ. Pharmacogenetics of warfarin: regulatory, scientific, and clinical issues. J Thromb Thrombolysis. 2008; 25(1):45-51. [PubMed: 17906972]

11. Gage BF, Eby C, Johnson JA, et al. Use of pharmacogenetic and clinical factors to predict the therapeutic dose of warfarin. Clin Pharmacol Ther. 2008; 84(3):326-31. [PubMed: 18305455]

12. Schulman S. Clinical practice. Care of patients receiving long-term anticoagulant therapy. N Engl J Med. 2003; 349(7):675-83. [PubMed: 12917305]

13. Holbrook AM, Pereira JA, Labiris R, et al. Systematic overview of warfarin and its drug and food interactions. Arch Intern Med. 2005; 165(10):1095-106. [PubMed: 15911722]

14. Jacobs LG. Warfarin pharmacology, clinical management, and evaluation of hemorrhagic risk for the elderly. Cardiology clinics. 2008; 26(2):157-67. v. [PubMed: 18406992]

15. Glasheen JJ, Fugit RV, Prochazka AV. The risk of overanticoagulation with antibiotic use in outpatients on stable warfarin regimens. J Gen Intern Med. 2005; 20(7):653-6. [PubMed: 16050864]

16. Visser LE, Penning-van Bees FJ, Kasbergen AA, et al. Overanticoagulation associated with combined use of antibacterial drugs and acenocoumarol or phenprocoumon anticoagulants. Thromb Haemost. 2002; 88(5):705-10. [PubMed: 12428081]

17. O'Reilly RA. The stereoselective interaction of warfarin and metronidazole in man. N Engl J Med. 1976; 295(7):354-7. [PubMed: 934223] 
18. Kunze KL, Wienkers LC, Thummel KE, Trager WF. Warfarin-fluconazole. I. Inhibition of the human cytochrome P450-dependent metabolism of warfarin by fluconazole: in vitro studies. Drug Metab Dispos. 1996; 24(4):414-21. [PubMed: 8801056]

19. Black DJ, Kunze KL, Wienkers LC, et al. Warfarin-fluconazole. II. A metabolically based drug interaction: in vivo studies. Drug Metab Dispos. 1996; 24(4):422-8. [PubMed: 8801057]

20. Israel DS, Stotka J, Rock W, et al. Effect of ciprofloxacin on the pharmacokinetics and pharmacodynamics of warfarin. Clinical infectious diseases : an official publication of the Infectious Diseases Society of America. 1996; 22(2):251-6. [PubMed: 8838180]

21. Lane G. Increased hypoprothrombinemic effect of warfarin possibly induced by azithromycin. Ann Pharmacother. 1996; 30(7-8):884-5. [PubMed: 8826581]

22. Woldtvedt BR, Cahoon CL, Bradley LA, Miller SJ. Possible increased anticoagulation effect of warfarin induced by azithromycin. Ann Pharmacother. 1998; 32(2):269-70. [PubMed: 9496419]

23. Recker MW, Kier KL. Potential interaction between clarithromycin and warfarin. Ann Pharmacother. 1997; 31(9):996-8. [PubMed: 9296238]

24. Udall JA. Human Sources and Absorption of Vitamin K in Relation to Anticoagulation Stability. JAMA: The Journal of the American Medical Association. 1965; 194(2):127-9. [PubMed: 5897315]

25. Hochman R, Clark J, Rolla A, et al. Bleeding in patients with infections. Are antibiotics helping or hurting? Arch Intern Med. 1982; 142:1440-2. [PubMed: 7103622]

26. Lane MA, Devine ST, McDonald JR. High-risk antimicrobial prescriptions among ambulatory patients on warfarin. Journal of clinical pharmacy and therapeutics. 2012; 37(2):157-60. [PubMed: 21517927]

27. Arnason T, Wells PS, van Walraven C, Forster AJ. Accuracy of coding for possible warfarin complications in hospital discharge abstracts. Thromb Res. 2006; 118(2):253-62. [PubMed: 16081144]

28. Baillargeon J, Holmes HM, Lin YL, et al. Concurrent Use of Warfarin and Antibiotics and the Risk of Bleeding in Older Adults. The American journal of medicine. 2012; 125(2):183-9. [PubMed: 22269622]

29. Penning-van Beest F, Erkens J, Petersen KU, et al. Main comedications associated with major bleeding during anticoagulant therapy with coumarins. Eur J Clin Pharmacol. 2005; 61:439-44. [PubMed: 15947920]

30. Linkins LA, Choi PT, Douketis JD. Clinical impact of bleeding in patients taking oral anticoagulant therapy for venous thromboembolism: a meta-analysis. Ann Intern Med. 2003; 139(11):893-900. [PubMed: 14644891]

31. Landefeld CS, Goldman L. Major bleeding in outpatients treated with warfarin: incidence and prediction by factors known at the start of outpatient therapy. The American journal of medicine. 1989; 87(2):144-52. [PubMed: 2787958]

32. Douketis JD, Foster GA, Crowther MA, et al. Clinical risk factors and timing of recurrent venous thromboembolism during the initial 3 months of anticoagulant therapy. Arch Intern Med. 2000; 160(22):3431-6. [PubMed: 11112236]

33. Fihn SD, McDonell M, Martin D, et al. Risk factors for complications of chronic anticoagulation. A multicenter study. Warfarin Optimized Outpatient Follow-up Study Group. Ann Intern Med. 1993; 118(7):511-20. [PubMed: 8280198]

34. Fischer HD, Juurlink DN, Mamdani MM, et al. Hemorrhage During Warfarin Therapy Associated With Cotrimoxazole and Other Urinary Tract Anti-infective Agents: A Population-Based Study. Arch Intern Med. 2010; 170(7):617-21. [PubMed: 20386005]

35. Schelleman H, Bilker WB, Brensinger CM, et al. Warfarin with fluoroquinolones, sulfonamides, or azole antifungals: interactions and the risk of hospitalization for gastrointestinal bleeding. Clin Pharmacol Ther. 2008; 84(5):581-8. [PubMed: 18685566]

36. Clark NP, Delate T, Riggs CS, et al. Warfarin Interactions With Antibiotics in the Ambulatory Care Setting. JAMA internal medicine. 2014

37. Altman R, Rouvier J, Gurfinkel E, et al. Comparison of two levels of anticoagulant therapy in patients with substitute heart valves. J Thorac Cardiovasc Surg. 1991; 101(3):427-31. [PubMed: 1999935] 
38. Hull R, Hirsh J, Jay R, et al. Different intensities of oral anticoagulant therapy in the treatment of proximal-vein thrombosis. N Engl J Med. 1982; 307(27):1676-81. [PubMed: 6755255]

39. Saour JN, Sieck JO, Mamo LA, Gallus AS. Trial of different intensities of anticoagulation in patients with prosthetic heart valves. N Engl J Med. 1990; 322(7):428-32. [PubMed: 2300106]

40. Turpie AG, Gunstensen J, Hirsh J, et al. Randomised comparison of two intensities of oral anticoagulant therapy after tissue heart valve replacement. Lancet. 1988; 1(8597):1242-5. [PubMed: 2897516]

41. Singer DE, Chang Y, Fang MC, et al. Should Patient Characteristics Influence Target Anticoagulation Intensity for Stroke Prevention in Nonvalvular Atrial Fibrillation? Circulation: Cardiovascular Quality and Outcomes. 2009; 2(4):297-304. [PubMed: 20031854]

42. Cunningham A, Stein CM, Chung CP, et al. An automated database case definition for serious bleeding related to oral anticoagulant use. Pharmacoepidemiology and drug safety. 2011 


\section{Clinical Significance}

- Patients who receive warfarin and a high-risk antibiotic are at increased risk for significant bleeding events.

- Alterations in the INR are common among patients who receive antibiotics

- INR evaluation within 3-14 days of antibiotic prescription may reduce the risk of serious bleeding events among patients receiving warfarin. 
Table 1

Demographic and Clinical Characteristics

\begin{tabular}{|c|c|c|c|c|}
\hline & Total & Low-Risk Antibiotic ${ }^{I}$ & High-Risk Antibiotic ${ }^{2}$ & P-value \\
\hline $\mathrm{N}$ & 22,722 & 8,194 & 14,078 & \\
\hline Mean Age (SD) & $69.5(10.8)$ & $68.8(11.1)$ & $69.8(10.7)$ & $<.0001$ \\
\hline \multicolumn{5}{|l|}{ Gender (\% male) } \\
\hline \multicolumn{5}{|l|}{ Race $(\%)$} \\
\hline White & $19,631(88.1)$ & $7,323(89.4)$ & $12,308(87.4)$ & $<.0001$ \\
\hline Black & $1,803(8.1)$ & $568(6.9)$ & $1,235(8.8)$ & $<.0001$ \\
\hline Other & $838(3.8)$ & $303(3.7)$ & $535(3.8)$ & 0.7 \\
\hline \multicolumn{5}{|l|}{ Comorbidities (\%) } \\
\hline Diabetes Mellitus & $9,930(44.6)$ & 3,739 (45.6) & $6,191(44.0)$ & 0.02 \\
\hline Hypertension & $19,592(88.0)$ & $7,268(88.7)$ & $12,324(87.5)$ & 0.01 \\
\hline Heart Failure & $9,701(43.6)$ & $3,518(42.9)$ & $6,183(43.9)$ & 0.2 \\
\hline Malignancy & $6,676(30.0)$ & $2,369(28.9)$ & $4,307(30.6)$ & 0.008 \\
\hline Chronic Lung Disease & $11,170(50.2)$ & $3,610(44.1)$ & $7,560(53.7)$ & $<.0001$ \\
\hline Renal Failure & $3,501(15.7)$ & $1,301(15.9)$ & $2,200(15.6)$ & 0.6 \\
\hline Liver Disease & $930(4.2)$ & $335(4.1)$ & $595(4.2)$ & 0.6 \\
\hline Ischemic Heart Disease & $14,745(66.2)$ & $5,395(65.8)$ & $9,350(66.4)$ & 0.4 \\
\hline Valvular Heart Disease & $5,663(25.4)$ & $2,135(26.1)$ & $3,528(25.1)$ & 0.1 \\
\hline Alcohol Dependence & $1,624(7.3)$ & $643(7.9)$ & $981(7.0)$ & 0.02 \\
\hline None of the Above & $423(1.9)$ & $170(2.1)$ & $253(1.8)$ & 0.1 \\
\hline \multicolumn{5}{|c|}{ Indication for Anticoagulation (\%) } \\
\hline Heart Valve Replacement & $4,325(19.4)$ & $1,672(20.4)$ & $2,653(18.9)$ & 0.005 \\
\hline Other & $19,113(85.8)$ & $7,028(85.8)$ & $12,085(85.8)$ & 0.9 \\
\hline Interacting Medications (\%) & $19,555(87.8)$ & $6,872(83.9)$ & $12,085(85.8)$ & $<.0001$ \\
\hline Distance $>20$ miles $(\%)$ & $12,288(55.2)$ & $4,415(53.9)$ & $7,873(55.9)$ & 0.003 \\
\hline Early INR Check $*(\%)$ & $9,770(43.8)$ & $3,504(42.8)$ & $6,266(44.5)$ & 0.01 \\
\hline
\end{tabular}

${ }^{1}$ Low-Risk Antibiotics: clindamycin, cephalexin

${ }^{2}$ High-Risk Antibiotics: trimethoprim/sulfamethoxazole (TMP/SMX), ciprofloxacin, levofloxacin, metronidazole, fluconazole, azithromycin and clarithromycin 
Table 2

Multivariate Predictors of Bleeding Event as Primary Diagnosis

\begin{tabular}{|c|c|c|c|}
\hline & (+) Bleeding Event & (-) Bleeding Event & HR $(95 \%$ CI $)$ \\
\hline $\mathrm{N}$ & 129 & 22,143 & - \\
\hline Mean Age (SD) & $71.2(9.8)$ & $69.5(10.8)$ & $1.00(0.99-1.02)$ \\
\hline Gender ( $\%$ male $)$ & $123(95.4)$ & $21,579(97.5)$ & $0.5(0.22-1.13)$ \\
\hline \multicolumn{4}{|l|}{ Race $(\%)$} \\
\hline White & $120(93.0)$ & $19,511(88.1)$ & Referent \\
\hline Black & $6(4.7)$ & $1,797(8.1)$ & $0.47(0.21-1.10)$ \\
\hline Other & $3(2.3)$ & $835(3.8)$ & $0.61(0.19-1.94)$ \\
\hline \multicolumn{4}{|l|}{ Comorbidities (\%) } \\
\hline Diabetes Mellitus & 67 (51.9) & $9,866(44.6)$ & $1.07(0.74-1.55)$ \\
\hline Hypertension & $124(96.1)$ & $19,470(87.9)$ & $2.15(0.87-5.31)$ \\
\hline Heart Failure & $78(60.5)$ & $9,629(43.5)$ & $1.21(0.82-1.80)$ \\
\hline Malignancy & $64(49.6)$ & $6,619(29.9)$ & $1.89(1.33-2.70)$ \\
\hline Chronic Lung Disease & $81(62.8)$ & $11,095(50.1)$ & $1.16(0.80-1.68)$ \\
\hline Renal Failure & $50(38.8)$ & $3,458(15.6)$ & $2.53(1.74-3.68)$ \\
\hline Liver Disease & $12(9.3)$ & $920(4.2)$ & $1.61(0.86-3.00)$ \\
\hline Ischemic Heart Disease & $99(76.7)$ & $14,648(66.2)$ & $0.83(0.60-1.44)$ \\
\hline Valvular Heart Disease & $55(42.6)$ & $5,611(25.3)$ & $1.34(0.90-1.98)$ \\
\hline Alcohol Dependence & $13(10.1)$ & $1,611(7.3)$ & $1.47(0.82-2.63)$ \\
\hline None of the Above & $0(0.0)$ & $423(1.9)$ & Referent \\
\hline \multicolumn{4}{|l|}{ Indication for Anticoagulation } \\
\hline Heart Valve Replacement & $51(39.5)$ & $4,275(19.3)$ & $2.12(1.43-3.14)$ \\
\hline Other & $116(89.9)$ & $19,000(85.8)$ & $1.30(0.71-2.38)$ \\
\hline Interacting Medications (\%) & $123(95.4)$ & $19,436(87.8)$ & $2.29(1.01-5.19)$ \\
\hline Distance $>20$ miles $(\%)$ & $74(57.4)$ & $12,216(55.2)$ & $1.15(0.80-1.64)$ \\
\hline Early INR Check ${ }^{*}(\%)$ & $48(37.2)$ & $9,714(43.9)$ & $0.61(0.42-0.88)$ \\
\hline High-Risk Antibiotic (\%) & $93(72.1)$ & $13,988(63.2)$ & $1.48(1.00-2.19)$ \\
\hline TMP/SMX & $14(10.9)$ & $1,964(8.9)$ & $1.79(0.97-3.32)$ \\
\hline Ciprofloxacin & $30(23.3)$ & $4,349(19.6)$ & $1.42(0.87-2.31)$ \\
\hline Levofloxacin & $11(8.5)$ & $2,521(11.4)$ & $1.30(0.66-2.55)$ \\
\hline Metronidazole & $5(3.9)$ & $755(3.4)$ & $1.63(0.61-4.39)$ \\
\hline Fluconazole & $3(2.3)$ & $287(1.3)$ & $2.11(0.60-7.34)$ \\
\hline Azithromycin & $33(25.6)$ & $5,725(25.9)$ & $1.93(1.13-3.30)$ \\
\hline Clarithromycin & $2(1.6)$ & $481(2.3)$ & $1.71(0.45-6.57)$ \\
\hline Co-prescribed High-risk & $8(6.2)$ & $991(4.5)$ & $1.75(0.79-3.89)$ \\
\hline Co-prescribed High- and Low- risk & $8(6.2)$ & $1,162(5.2)$ & $1.35(0.61-2.99)$ \\
\hline Low-Risk Antibiotic (\%) & $36(27.9)$ & $8,155(36.8)$ & Referent \\
\hline Clindamycin & $11(8.5)$ & $1,862(8.4)$ & $0.58(0.31-1.09)$ \\
\hline Cephalexin & $34(26.4)$ & $6,561(29.6)$ & $0.07(0.48-1.04)$ \\
\hline
\end{tabular}

INR = International Normalized Ratio 
TMP/SMX $=$ Trimethoprim/Sulfamethoxazole

* INR evaluation within 3-14 days of antibiotic co-prescription 
Table 3

Alterations in INR Following Antibiotic Administration

\begin{tabular}{lcccc}
\hline & Total & $\mathbf{\leq 4}$ & Peak INR >4 - $\leq \mathbf{6}$ & $\mathbf{> 6}$ \\
\hline High-risk Antibiotic (\%) & 22,381 & $20,061(89.6)$ & $1,748(7.8)$ & $572(2.6)$ \\
TMP/SMX & 2,828 & $2,538(89.7)$ & $227(8.0)$ & $63(2.2)$ \\
Ciprofloxacin & 6,844 & $6,195(90.5)$ & $514(7.5)$ & $135(2.0)$ \\
Levofloxacin & 3,343 & $2,972(88.9)$ & $279(8.4)$ & $92(2.8)$ \\
Metronidazole & 1,003 & $853(85.0)$ & $101(10.1)$ & $49(4.9)$ \\
Fluconazole & 381 & $291(76.4)$ & $53(13.9)$ & $37(9.7)$ \\
Azithromycin & 9,331 & $8,526(91.4)$ & $649(7.0)$ & $156(1.6)$ \\
Clarithromycin & 584 & $521(89.2)$ & $46(7.9)$ & $17(2.9)$ \\
Co-prescribed High-risk & 1,357 & $1,153(85.0)$ & $151(11.1)$ & $53(4.0)$ \\
Co-prescribed High- and Low-risk & 2,401 & $2,089(87.0)$ & $226(9.4)$ & $86(3.6)$ \\
Low-risk Antibiotic (\%) & 12,973 & $11,799(91.0)$ & $931(7.2)$ & $243(2.9)$ \\
\hline
\end{tabular}

INR = International Normalized Ratio

TMP/SMX = Trimethoprim/Sulfamethoxazole 